\title{
Electronic and magnetic properties of the cation vacancy defect in $m-\mathrm{HfO}_{2}$
}

\author{
Keith P. McKenna ${ }^{1, *}$ and David Muñoz Ramo ${ }^{2, \dagger}$ \\ ${ }^{1}$ Department of Physics, University of York, Heslington, York YO10 5DD, United Kingdom \\ ${ }^{2}$ Department of Chemistry, University of Cambridge, Lensfield Road, Cambridge CB2 1EW, United Kingdom
}

(Received 26 August 2015; published 23 November 2015)

\begin{abstract}
The electronic and magnetic properties of cation vacancies in $m-\mathrm{HfO}_{2}$ are predicted using density functional theory. The hafnium vacancy is found to introduce a series of charge transition levels in the range $0.76-1.67 \mathrm{eV}$ above the valence band maximum associated with holes localized on neighboring oxygen sites. The neutral defect adopts a $S=2$ spin state, and we compute corresponding $g$ tensors to aid electron experimental identification of the defect by electron spin resonance spectroscopy. We find that separated vacancies exhibit weak ferromagnetic coupling and the interaction is highly anisotropic - being much stronger when mediated by planes of threecoordinated oxygen ions. Further, we characterize the process of thermal detachment of a hole from a neutral vacancy providing an atomistic model for the $p$-type conductivity observed experimentally at high temperature. These results provide invaluable information on the electronic and magnetic properties of cation vacancies in $\mathrm{HfO}_{2}$ and can aid future experimental identification of these complex defects.
\end{abstract}

DOI: 10.1103/PhysRevB.92.205124

PACS number(s): 75.50.Pp, 71.55.-i, 71.38.Ht

\section{INTRODUCTION}

Vacancies are one of the most commonly occurring defects in metal oxide materials and are responsible for diverse optoelectronic phenomena of both fundamental and practical significance. For example, their presence is responsible for modifying the optical properties of minerals [1-3], intrinsic electrical conductivity in semiconducting oxides [4-8], charge trapping in microelectronic devices [9-13], and electronhole recombination centers in photovoltaic or photocatalytic materials [14-16]. Oxygen vacancy defects have received considerable attention since many natural and synthesized metal oxide materials are oxygen deficient. While cation vacancies are also usually present, their concentration and properties have been characterized for very few materials [17-23]. On one hand, this is because widely used experimental probes such as absorption and luminescence spectroscopy and deep level transient spectroscopy cannot easily discriminate cation vacancy defects from other intrinsic and extrinsic defects that may be present in materials. On the other hand, first principles theoretical methods that have proved invaluable for isolating and resolving the properties of oxygen vacancy defects are challenging to apply due to errors associated with the presence of localized holes [24,25]. The uncertainty that remains has fueled considerable speculation on the possible role of cation vacancies in oxides. One such example is provided by hafnium dioxide $\left(\mathrm{HfO}_{2}\right)$, a material which finds a number of applications in microelectronics [26,27]. It has been suggested that cation vacancies can induce high temperature $p$-type conductivity [28-30] and contribute to the so far unexplained ferromagnetism in $\mathrm{HfO}_{2}$ [31-35]. However, our knowledge

\footnotetext{
*keith.mckenna@york.ac.uk

†dm586@cam.ac.uk
}

Published by the American Physical Society under the terms of the Creative Commons Attribution 3.0 License. Further distribution of this work must maintain attribution to the author(s) and the published article's title, journal citation, and DOI. of the fundamental electronic and magnetic properties of cation vacancies in $\mathrm{HfO}_{2}$ remains extremely limited, presenting an obstacle to deeper understanding of these complex effects.

In this paper we employ density functional theory (DFT) to predict the electronic and magnetic properties of cation vacancies in monoclinic hafnium dioxide $\left(m-\mathrm{HfO}_{2}\right)$ from first principles. To ensure accuracy of the results we employ two different approaches that eliminate, at least in part, the self-interaction (SI) error present in widely used DFT approximations $[24,25]$. We show that these two SI corrected approaches predict properties that are very consistent with each other, giving us confidence in the results. On the other hand, standard semilocal approximations to exchange and correlation yield a qualitatively incorrect description of the defect. We show that the neutral hafnium vacancy involves four holes localized on neighboring oxygen sites associated with a series of charge transition levels in the range 0.76 $1.67 \mathrm{eV}$ above the valence band maximum. The defect is predicted to exhibit a net magnetic moment of $4 \mu_{\mathrm{B}}$, being $20 \mathrm{meV}$ more stable than the zero moment state. However, the ferromagnetic interaction between separated vacancies is very weak even at high concentrations due to the localized nature of the holes suggesting cation vacancies are an unlikely candidate for explaining the observed ferromagnetism. We find that although in the ground state four holes are localized near the neutral vacancy, one of the holes is relatively weakly bound and can detach as a free polaron providing an atomistic model for the observed $p$-type conductivity at high temperature [28-30]. We compute $g$ tensors associated with the cation vacancy defect to aid electron experimental identification of the defect by electron spin resonance (ESR) spectroscopy and also discuss implications of the predictions for other experimental probes such as absorption spectroscopy. Altogether, these results provide deep insight into the electronic and magnetic properties of cation vacancies in $\mathrm{HfO}_{2}$ and will aid future experimental identification of these complex defects.

The rest of the paper is organized as follows. In Sec. II we discuss previous experimental and theoretical studies on cation 
vacancy defects in oxides as well as the challenges involved in theoretical prediction of their properties. In Sec. III we present results on the electronic and magnetic properties of cation vacancies in $\mathrm{HfO}_{2}$. Finally, in Secs. IV and V we discuss the results and present our main conclusions.

\section{BACKGROUND}

Cation vacancies in most metal oxide materials are able to trap one or a number of holes. In many cases these holes are found to localize on individual oxygen ions surrounding the vacancy forming $\mathrm{O}^{-}$ions $[20,23,25]$. The localization of holes in this way in many cases breaks the point group symmetry of the defect through an asymmetric lattice distortion. Therefore, such a defect is perhaps better considered as an acceptor defect with a number of bound small hole polarons [20]. Cation vacancies of this type are thought to exist in most metal oxide materials, but relatively few materials have been well characterized experimentally. A good example is the $\mathrm{V}^{-}$center in $\mathrm{MgO}$ which consists of a missing $\mathrm{Mg}^{2+}$ ion with a single hole localized on a neighboring oxygen ion. The localization of the hole has been detected directly for this defect by ESR spectroscopy $[18,19]$. An associated optical absorption band peaking near $2.3 \mathrm{eV}$ has also been identified, which is attributed to electronic transitions to the unoccupied hole state above the valence band maximum [17,20]. There is experimental evidence that similar defects exist in a wider range of metal oxide materials including $\mathrm{CaO}$ [36], $\mathrm{NiO}$ [37], $\mathrm{ZnO}$ [38], $\mathrm{TiO}_{2}$ [39], $\mathrm{HfO}_{2}$ [28-30], and a number of perovskites [40], but in most cases the understanding of associated electronic properties is incomplete.

In the absence of clear experimental information, first principles calculations of defect properties using DFT can be invaluable. However, modeling the structure and properties of cation vacancy defects using DFT remains extremely challenging because common semilocal approximations to the exchange-correlation functional, such as the generalized gradient approximation (GGA), suffer from SI error which tends to artificially delocalize holes [24,25]. Nevertheless there are numerous examples of GGA-DFT calculations for cation vacancies in oxides which generally predict very shallow hole states at odds with experiment [41-43]. Such defects are often predicted to lack any symmetry breaking polaronic distortion, even for defects which are known to adopt a lower symmetry structure such as the $\mathrm{V}^{-}$center in $\mathrm{MgO}$ [16]. Predictions regarding the magnetic interaction between cation defects are also likely to be inaccurate due to the artificially delocalized hole states which will exhibit a longer range direct exchange interaction with other holes. Approaches such as DFT + $U$ [44], cancellation of nonlinearity [25,45], and hybrid DFT functionals incorporating nonlocal exchange [8,46,47] eliminate at least part of the SI error and so offer a route to more accurate prediction of cation vacancy properties. Many of these approaches involve a parameter which controls the strength of SI correction (e.g., $U$ in DFT $+U$ or the percentage of Hartree-Fock exchange in hybrid functionals) which in principle can be determined unambiguously by ensuring that the correct linear behavior of the total energy with respect to fractional occupation number is obtained. However, there are still relatively few examples where SI corrected approaches have been used to model the properties of cation vacancy defects in oxides. These include vacancies in $\mathrm{MgO}$ [23], $\mathrm{NiO}$ [7], $\mathrm{Cu}_{2} \mathrm{O}$ [21,48], $\mathrm{ZnO}$ [49], and several mixed oxides with the spinel structure [50].

Relatively little is known experimentally about the properties of cation vacancies in $\mathrm{HfO}_{2}$. There is some experimental evidence pointing to the fact that they may serve as donors of mobile holes at high temperatures leading to $p$-type conductivity [28-30]. Early studies on the temperature dependence of conductivity in $\mathrm{HfO}_{2}$ identified two regimes of $p$-type conductivity. At low temperatures hole conduction was found to be associated with an activation energy of $0.7 \mathrm{eV}$, whereas at higher temperatures the associated activation energy was significantly reduced. This was interpreted in terms of the thermal liberation of holes from cation vacancies, i.e., $\mathrm{V}_{\mathrm{Hf}}^{\times} \rightarrow \mathrm{V}_{\mathrm{Hf}}^{\prime}+h$ [28,29]. This idea is corroborated by more recent results which also identify cation vacancies as a source of high-temperature $p$-type conductivity in $\mathrm{HfO}_{2}$ [30]. In a previous theoretical study we showed how the activation energy for small hole polaron diffusion in $m-\mathrm{HfO}_{2}$ is rather low, $0.14 \mathrm{eV}$, consistent with the high temperature conductivity observations [51]. There has also been some speculation on the possible role cation vacancies may play in explaining observed ferromagnetism [31-33,35]. However, DFT calculations for $m-\mathrm{HfO}_{2}$ employing local or semilocal functionals found relatively weak ferromagnetic coupling between cation vacancies insufficient to explain experimental observations [34,52].

\section{METHODS}

Density functional theory (DFT) calculations are performed using the projector augmented wave method as implemented within the Vienna $a b$ initio simulation package $[53,54]$. The $5 p, 6 s$, and $5 d$ electrons of $\mathrm{Hf}$ and the $2 s$ and $2 p$ electrons of $\mathrm{O}$ are treated as valence electrons and expanded in a plane wave basis with energies up to $300 \mathrm{eV}$ (400 eV for cell optimization). For the conventional cell of monoclinic $\mathrm{HfO}_{2}$, which is the most stable phase in ambient conditions, an $8 \times 8 \times 8$ Monkhorst-Pack $k$-point grid is used, and structural optimization is performed until forces are less than $0.01 \mathrm{eV} / \AA$. Using the Perdew-Burke-Ernzerhof (PBE) exchange correlation functional we obtain lattice parameters within $0.6 \%$ of experiment for $m-\mathrm{HfO}_{2}(a=5.142 \AA, b=$ $5.192 \AA, c=5.250 \AA$, and $\beta=99.65^{\circ}$ ).

To correct the SI error in the PBE exchange correlation functional we employ the cancellation of nonlinearity (CON) method [25,45] that has been demonstrated previously for modeling small hole polarons in $\mathrm{HfO}_{2}[51,55]$. This method has also been successfully applied previously to model a range of hole centers in oxides including acceptor defects in transparent conducting oxides $[45,56]$. The CON approach applies a local occupation dependent potential to the oxygen $p$ states of the following form:

$$
V_{\mathrm{hs}}=\lambda_{\mathrm{hs}}\left(1-n_{m, \sigma} / n_{\text {host }}\right),
$$

where $n_{m, \sigma}$ is the fractional occupancy of sublevel $m$ of spin $\sigma$ in the oxygen $p$ orbital. The reference occupation $n_{\text {host }}$ is the anion $p$-orbital occupancy in the absence of holes, as determined from the neutral defect-free system. The only free parameter in the potential, $\lambda_{\mathrm{hs}}$, is determined by ensuring that 
the correct linear behavior of the total energy with respect to fractional occupation number is obtained [25,57]. In a previous paper we described the parametrization of this approach for hole polarons in $\mathrm{HfO}_{2}$ yielding $\lambda_{\mathrm{hs}}=3.8 \mathrm{eV}$ [51].

Defect calculations for the PBE and CON approximations are performed using a $3 \times 3 \times 3$ supercell (324 atoms). We also perform calculations using the Heyd, Scuseria, and Ernzerhof (HSE) hybrid functional which includes nonlocal exchange in order to correct the SI error [46]. Using the HSE functional we obtain lattice parameters for $m-\mathrm{HfO}_{2}$ again in good agreement with experiment ( $a=5.144 \AA, b=5.190 \AA$, $c=5.330 \AA$, and $\left.\beta=99.66^{\circ}\right)$. Defect calculations for the HSE functional are performed using a smaller $2 \times 2 \times 2$ supercell (96 atoms) owing to the increased computation expense of these calculations. In all cases total energies are corrected for potential alignment and image-charge interactions as described in Ref. [25].

The ESR properties of the Hf vacancy in its different charge states are calculated by means of an embedded cluster model described in detail in Refs. [58] and [59]. This model has been used successfully to calculate the optical properties and ESR parameters of oxygen vacancies, electron and hole polarons, and excitons in pure and doped $m$-HfO2 [47,58,60,61]. In this model, a quantum-mechanical (QM) cluster including the vacancy, the distorted region accommodating the defect, and its vicinity is embedded into the rest of the crystal represented by a lattice of classical rigid ions. In order to design this model, we construct an approximately spherical nanocluster using as a building block a 324-atom supercell of bulk $m-\mathrm{HfO}_{2}$ with zero charge and dipole moment, with its geometry obtained from the periodic DFT calculations. This nanocluster contains 8748 classical ions and has a radius of about $26 \AA$. Then we create the vacancy environment by removing one $\mathrm{Hf}$ atom in the center of the nanocluster and modifying the local geometry around the defect to exactly match the lattice relaxation obtained in the periodic calculations up to a radius of about $5 \AA$ (displacements of ions induced by the defect beyond this distance calculated in the periodic model are negligibly small). This computational scheme is implemented in the GUESS computer code, [59] which employs the GUASSIAN09 package [62] for calculating the electronic structure of the QM cluster in the electrostatic potential of the rest of the lattice.

The QM cluster surrounding the Hf vacancy includes 33 hafnium ions and 68 oxygen ions. All Hf ions outside the quantum cluster and within the radius of $11 \AA$ from the center are represented by large-core Hay and Wadt relativistic effective core potentials [63], which substitute all but four electrons of a hafnium atom. This prevents an artificial polarization of the electron density toward positive point ions outside the quantum cluster. The point ions outside the quantum cluster carry formal charges and contribute to the electrostatic potential on the quantum cluster ions (see Refs. [59,61], and [64] for more detail). As in previous studies, we use Gaussian basis sets on oxygen and hafnium ions optimized for the $m-\mathrm{HfO}_{2}$ case (see Ref. [65] for further details on the basis sets used). We use the HSE density functional to calculate the electronic structure and the $g$ tensor for the vacancy environment.

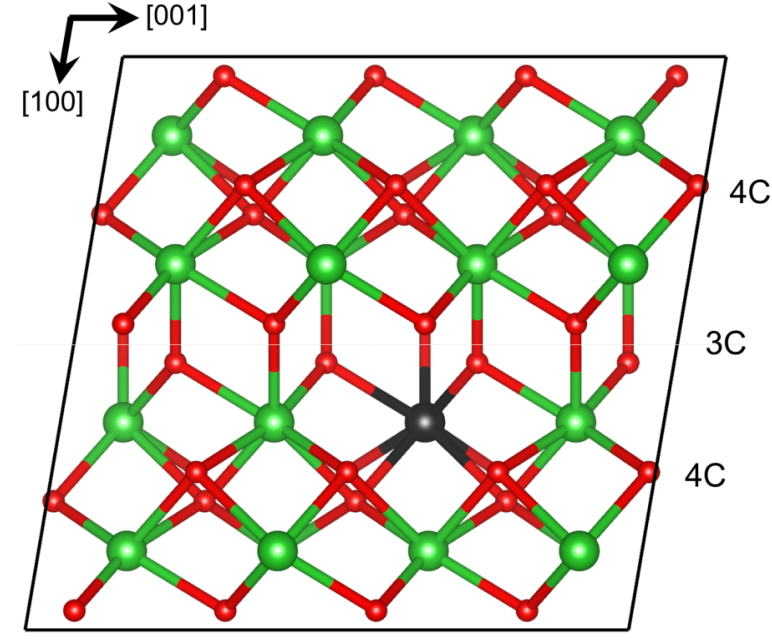

FIG. 1. (Color online) Crystal structure of $m-\mathrm{HfO}_{2}$ highlighting the layered nature of the three- and four-coordinated (3C and 4C) oxygen sublattices. A particular Hf site with its neighboring oxygen ions is highlighted (black). Large green spheres and small red spheres represent $\mathrm{Hf}$ and $\mathrm{O}$ ions, respectively.

\section{RESULTS}

\section{A. Formation energies}

The $m-\mathrm{HfO}_{2}$ crystal structure is characterized by two types of oxygen ion which differ in their coordination to hafnium ions, either three-coordinated (3C) or four-coordinated (4C). The hafnium ions are each coordinated to seven oxygen ions, three $3 \mathrm{C}$ and four $4 \mathrm{C}$. Viewed in the [010] projection, the $3 \mathrm{C}$ and $4 \mathrm{C}$ oxygen ions are arranged in alternating twodimensional layers separated by layers of hafnium (Fig. 1). Previous calculations using the B3LYP, CON, and HSE approaches have shown that hole polarons localize on both 3C and 4C oxygen sites with the former being more stable by about $0.4 \mathrm{eV}$ [47,51]. This effect is explained by the difference in electrostatic potential between the sites and different strain energy associated with polaronic distortion [51].

The bare cation vacancy that is created by removing a positive hafnium ion from the lattice has a formal charge of -4 . A range of other charge states are also possible corresponding to localization of holes on anions adjacent to the vacancy. In Kröger-Vink notation the defects we consider are $\mathrm{V}_{\mathrm{Hf}}^{\prime \prime \prime}, \mathrm{V}_{\mathrm{Hf}}^{\prime \prime \prime}, \mathrm{V}_{\mathrm{Hf}}^{\prime \prime}, \mathrm{V}_{\mathrm{Hf}}^{\prime}$, and $\mathrm{V}_{\mathrm{Hf}}^{X}$, corresponding to localization of zero, one, two, three, or four holes near the vacancy. To characterize the stability of such defects we calculate the defect formation energy,

$$
E_{f}\left(\left\{\mu_{i}\right\}, E_{\mathrm{F}}\right)=E_{\text {def }}^{q}-E_{\text {ideal }}+\sum_{i} \Delta n_{i} \mu_{i}+q E_{\mathrm{F}},
$$

where $E_{\mathrm{def}}^{q}$ is the total energy of the supercell containing a defect in relative charge state $q$, and $E_{\text {ideal }}$ is the total energy of the ideal bulk supercell. $\Delta n_{i}$ is the difference between the number of atoms of species $i$ in the defective and ideal supercells, $\mu_{i}$ is the chemical potential of species $i$, and $E_{\mathrm{F}}$ is the electron Fermi energy.

Defect formation energies are calculated at the CON, HSE, and GGA levels of theory using $\mu_{\mathrm{O}}=E\left(\mathrm{O}_{2}\right) / 2$ (i.e., half the 

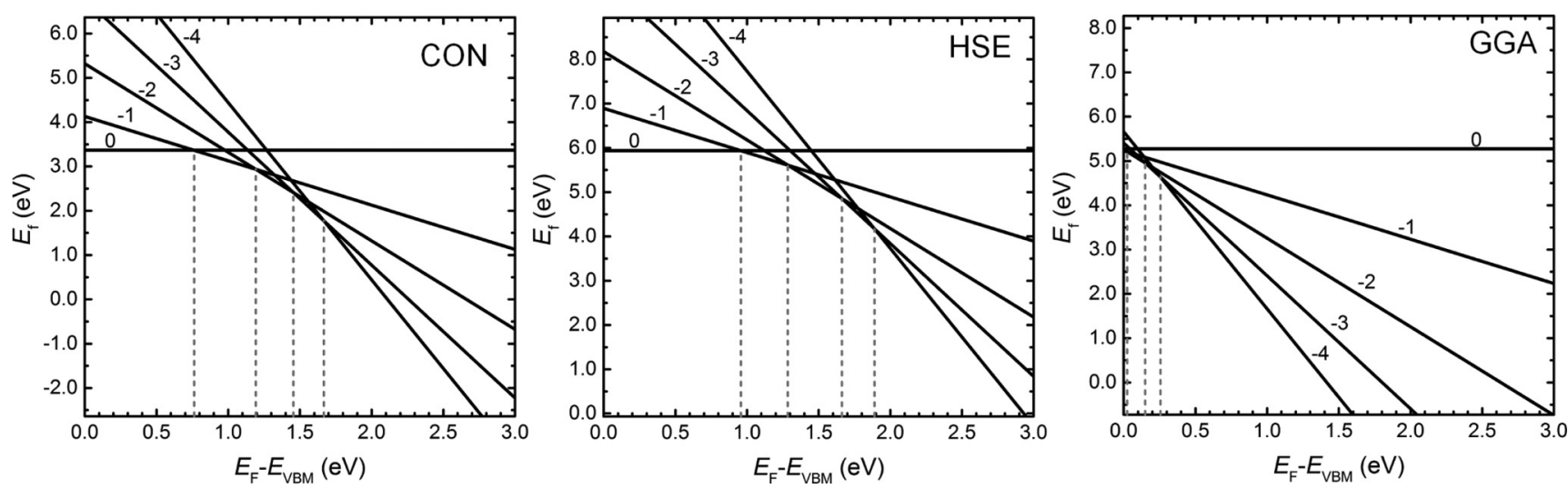

FIG. 2. Formation energy of $\mathrm{Hf}$ vacancy defects in $m-\mathrm{HfO}_{2}$ calculated using the CON, HSE, and GGA approaches. The formal charge of the defects and the charge transition levels (dotted lines) are also indicated.

energy of an oxygen molecule) corresponding to oxygen rich conditions (Fig. 2). Once $\mu_{\mathrm{O}}$ is fixed the chemical potential of $\mathrm{Hf}$ is also defined since $\mu_{\mathrm{HfO}_{2}}=\mu_{\mathrm{Hf}}+2 \mu_{\mathrm{O}}$. From these results one can read off equilibrium charge transition levels (CTLs) which are defined as the Fermi energy for which two defect charge states $q$ and $q^{\prime}$ have equal formation energy. We note that since the $\mathrm{CON}$ approach is not designed to describe $\mathrm{O}_{2}$, it leads to significant underbinding of the oxygen molecule. The consequence of this is that absolute formation energies are significantly underestimated, for example when compared to HSE. Similar effects have been found previously in DFT $+U$ calculations, and one approach to remedy the issue is to add a constant correction term to the oxygen chemical potential through comparison to experiment or high level quantum mechanical calculations [66]. Any such correction will only lead to a uniform vertical shift of the formation energies shown in Fig. 2 and will have no effect on the predicted CTLs. Since the main focus of this study is on electronic and magnetic properties, we do not attempt such a correction here. The SI corrected CON approach predicts a series of CTLs in the range $0.76-1.67 \mathrm{eV}$ above the valence band maximum. This prediction is also consistent with the results obtained using the HSE method (0.96-1.89 eV). GGA, on the other hand, predicts a series of CTLs in the range $0-0.26 \mathrm{eV}$, with the charge neutral defect being marginally unstable for all positive Fermi energies. This prediction, which is at odds with the known tendency of holes to form small polarons in $m-\mathrm{HfO}_{2}$, is a direct result of the SI error.

\section{B. Electronic properties}

The neutral Hf vacancy has a $S=2$ ground state associated with four holes which are localized on neighboring anion sites. The number of holes associated with the Hf vacancy defects depends on its charge. The optimized structure of each of the defects carrying a net spin along with isosurfaces of electron spin density are shown in Fig. 3. For the $\mathrm{V}_{\mathrm{Hf}}^{X}$ defect three holes are localized on $3 \mathrm{C}$ oxygen sites neighboring the vacancy with a fourth localized on a $4 \mathrm{C}$ oxygen site. The preference for hole trapping on the $3 \mathrm{C}$ sites is consistent with the increased stability of hole polarons on this sublattice. For the $\mathrm{V}_{\mathrm{Hf}}^{\prime}$ defect, the addition of an electron eliminates the hole on the least stable $4 \mathrm{C}$ oxygen site. Addition of subsequent electrons leading to the $\mathrm{V}_{\mathrm{Hf}}^{\prime \prime}, \mathrm{V}_{\mathrm{Hf}}^{\prime \prime \prime}$, and $\mathrm{V}_{\mathrm{Hf}}^{\prime \prime \prime \prime}$ defects eliminates the remaining holes one-by-one reducing the total spin of the defect in steps of $1 / 2$. The holes are removed in order of their distance from the Hf vacancy (furthest away first) consistent with the stabilizing electrostatic interaction between the positive hole and the $q=$ -4 charged vacancy.

The calculated electronic density of states for the $\mathrm{Hf}$ vacancy defects are shown in Fig. 4. The curves are aligned with respect to the bulk valence band maximum using the average electrostatic potential over ions far from the defect in different supercells as a common reference. The $\mathrm{V}_{\mathrm{Hf}}^{X}$ defect is associated with four localized electronic states in the gap between 1.5 and $2.3 \mathrm{eV}$ above the valence band. The eigenfunction associated with each electronic state in the gap is associated with a localized hole on one of the anions. As electrons are added the lowest unoccupied states are eliminated. In the case of the $\mathrm{V}_{\mathrm{Hf}}^{\prime}$ and $\mathrm{V}_{\mathrm{Hf}}^{\prime \prime}$ defects the occupied electron states drop below the valence band maximum meaning the highest occupied electronic states have a bulklike character. However for the $\mathrm{V}_{\mathrm{Hf}}^{\prime \prime \prime}$ and $\mathrm{V}_{\mathrm{Hf}}^{\prime \prime \prime \prime}$ defects the occupied states appear slightly above the bulk valence band maximum. The band gap
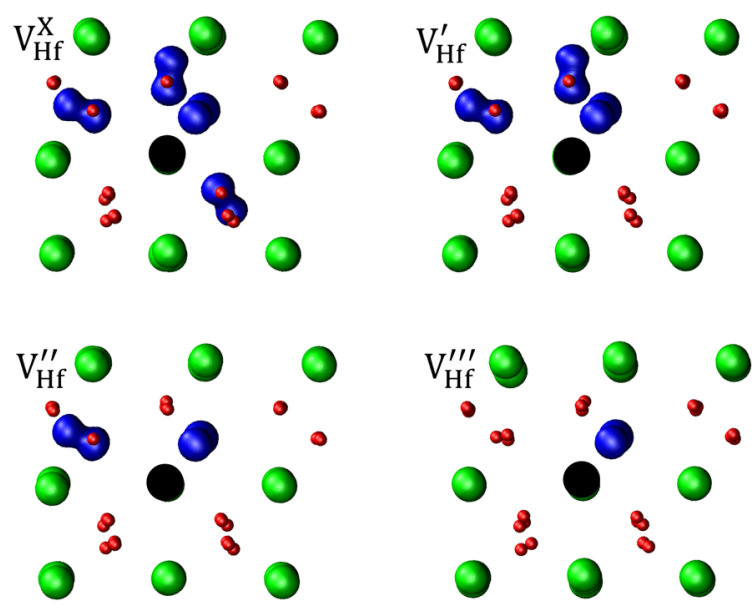

FIG. 3. (Color online) Optimized structure of the $\mathrm{V}_{\mathrm{Hf}}^{X}, \mathrm{~V}_{\mathrm{Hf}}^{\prime \prime}, \mathrm{V}_{\mathrm{Hf}}^{\prime \prime \prime}$, and $\mathrm{V}_{\mathrm{Hf}}^{\prime \prime \prime \prime}$ defects in $m-\mathrm{HfO}_{2}$. Large green spheres and small red spheres represent $\mathrm{Hf}$ and $\mathrm{O}$ ions, respectively, and the black circle indicates the location of the missing $\mathrm{Hf}$ ion. Electron spin density isosurfaces are shown in blue. 


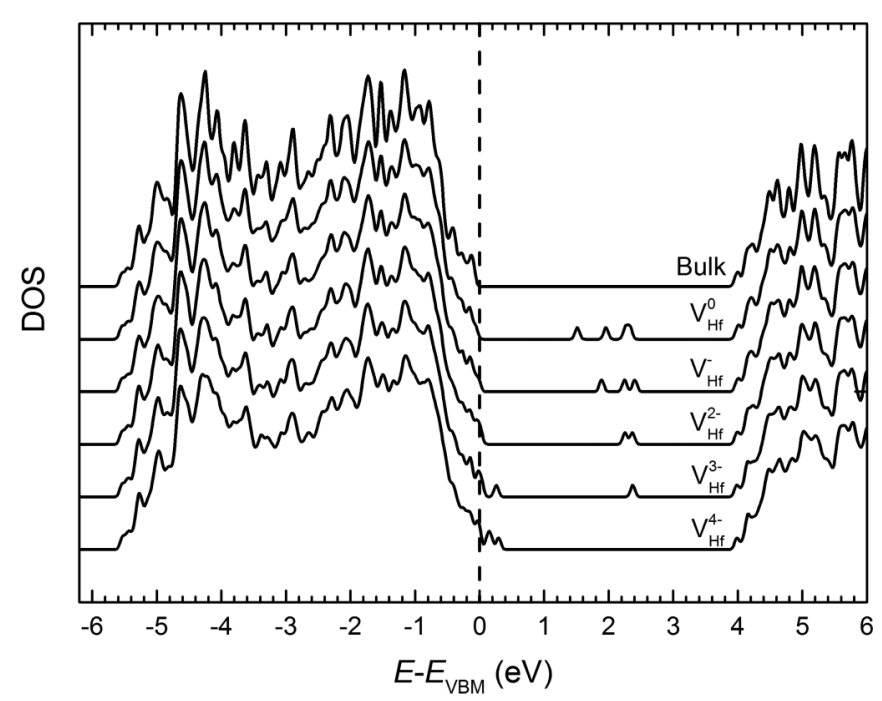

FIG. 4. Electronic density of states of the Hf vacancy defects in $\mathrm{HfO}_{2}$ calculated using the $\mathrm{CON}$ method. The curves are aligned with respect to the bulk valence band maximum and offset vertically for clarity.

within the CON approach is the same as within GGA and is underestimated with respect to experiment. However, the HSE calculations indicate that the position of the hole levels maintains the same relative position, but the gap increases by $1.6 \mathrm{eV}$ in much better agreement with experiment. The observation that the electronic properties of hole defects can be described accurately using the $\mathrm{CON}$ approach even though the band gap is underestimated reflects that the defect levels are derived from the valence band rather than the conduction band.

\section{Thermal detachment of holes}

The predicted strong localization of holes producing deep electronic states in the gap would seem to be at odds with the experimental evidence that cation vacancies can give rise to $p$-type conductivity (Sec. II). To assess the possibility that at elevated temperatures holes may detach from cation vacancies and become free small polarons, we perform calculations of the energetics associated with the process using the CON approach. We consider different initial guesses for the atomic structure and charge density in order that self-consistent minimization of the total energy yields a number of different metastable hole configurations. In this way we have identified the energetically favored pathway for detachment of a hole from a neutral vacancy. Figure 5 shows the spin density following diffusion of a single hole neighboring the vacancy from a $4 \mathrm{C}$ site to a nearby $3 \mathrm{C}$ site. The change in energy associated with this transformation is $0.34 \mathrm{eV}$. We also find that complete separation of this hole from the vacancy requires only a further $0.24 \mathrm{eV}(0.58 \mathrm{eV}$ in total). This small binding energy is a result of the high dielectric constant of hafnia as well as the energy gained by localizing the hole on the preferred $3 \mathrm{C}$ site rather than a 4C site. Subsequent detachment of holes from the $\mathrm{V}_{\mathrm{Hf}}^{\prime}$ defect costs an increasing amount of energy (starting from $1.01 \mathrm{eV}$ for the first hole and increasing to $1.49 \mathrm{eV}$ for the last). This suggests that under realistic conditions each vacancy can

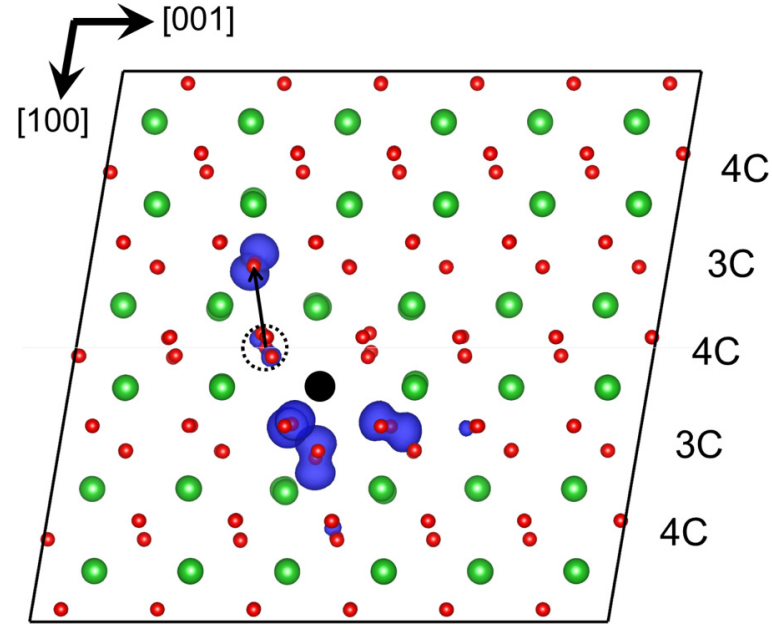

FIG. 5. (Color online) Diffusion of a hole from a neutral cation vacancy in $m-\mathrm{HfO}_{2}$ forming a negatively charged vacancy and a hole polaron on a $3 \mathrm{C}$ oxygen site. The black circle indicates the location of the missing $\mathrm{Hf}$ ion, and the dotted circle indicates the position of the hole before diffusion. Large green spheres and small red spheres represent $\mathrm{Hf}$ and $\mathrm{O}$ ions, respectively. Electron spin density isosurfaces are shown in blue.

provide on average one hole carrier rather than the four that might be expected on the basis of the formal ionic charge.

\section{Magnetic properties}

The presence of a net magnetic moment means cation vacancies may be amenable to identification by ESR spectroscopy. To assess the stability of this magnetic moment we also compute the energy of the most stable antiferromagnetic configuration in which two of the holes have their spin aligned antiparallel to the other two. We find this $S=0$ spin state is only $20 \mathrm{meV}$ less stable, suggesting the high spin state should be stable at low temperatures. To aid possible characterization we calculate the ESR $g$ tensors for cation vacancies using an embedded cluster approach as described in Sec. III. As discussed in the previous section, $V_{\mathrm{Hf}}^{X}$ and $\mathrm{V}_{\mathrm{Hf}}^{\prime}$ are the most likely charge states of cation vacancies in $m-\mathrm{HfO}_{2}$, and so we focus our attention on these defects. The principal components of the $g$ tensors are shown in Table I together with $g$ tensors for other spin defects in $m$ - $\mathrm{HfO}_{2}$ calculated previously using a similar approach $[47,58]$. The magnitude of the $g$-tensor components for cation vacancies are significantly larger and more anisotropic than any other defect, suggesting they could be clearly discriminated by ESR spectroscopy. The defects with the most similar $g$ tensors are those associated with small hole polarons as expected. The much higher anisotropy is consistent with the much lower symmetry of the cation vacancy defects compared to hole polarons. It is also notable that there is a significant difference between the $g$ tensor of the neutral and negatively charged vacancy reflecting their very different electronic structure and spin density [e.g., see Figs. 3(a), 3(b), and 4].

We also employ the CON approach to assess the strength of magnetic coupling between separated cation vacancies in $\mathrm{HfO}_{2}$. As shown in Fig. 6 we consider two neutral cation 
TABLE I. Principal components of the ESR $g$ tensors for the $\mathrm{V}_{\mathrm{Hf}}^{X}$ and $\mathrm{V}_{\mathrm{Hf}}^{\prime}$ defects. Previously calculated ESR $g$ tensors for other spin defects in $m-\mathrm{HfO}_{2}$ are also included including small hole polarons, the electron polaron, and oxygen vacancies on both oxygen sublattices $[47,58]$.

\begin{tabular}{lccc}
\hline \hline Defect & $g_{1}$ & $g_{2}$ & $g_{3}$ \\
\hline $\mathrm{V}_{\mathrm{Hf}}^{X}$ & 2.221 & 2.273 & 2.497 \\
$\mathrm{~V}_{\mathrm{Hf}}^{\prime}$ & 2.098 & 2.168 & 2.279 \\
$\mathrm{~V}_{\mathrm{O}}^{\bullet}(3 \mathrm{C})$ & 1.927 & 1.938 & 1.960 \\
$\mathrm{~V}_{\mathrm{O}}^{\bullet}(4 \mathrm{C})$ & 1.945 & 1.963 & 1.984 \\
$\mathrm{~V}_{\mathrm{O}}^{\prime}(4 \mathrm{C})$ & 1.811 & 1.845 & 1.941 \\
$\mathrm{O}_{\mathrm{O}}^{\bullet}(3 \mathrm{C})$ & 2.014 & 2.025 & 2.040 \\
$\mathrm{O}_{\mathrm{O}}^{\bullet}(4 \mathrm{C})$ & 2.009 & 2.037 & 2.043 \\
$\mathrm{Hf}$ & 1.812 & 1.829 & 1.922 \\
\hline \hline
\end{tabular}

vacancies separated by $8.3 \AA$ in the [001] direction (i.e., parallel to the $3 \mathrm{C}$ oxygen planes). Since the defects are treated in periodic boundary conditions, this system corresponds to a one-dimensional chain of vacancies. The ground state is found to be ferromagnetically aligned, and the energy to switch to antiferromagnetic alignment $E_{\mathrm{FM} / \mathrm{AFM}}=42 \mathrm{meV}$, giving an exchange constant of $J=E_{\mathrm{FM} / \mathrm{AFM}} / 4=10 \mathrm{meV}$. However, for defects separated by a similar distance in the [100] direction (perpendicular to the 3C layers) the exchange constant is reduced to $J=0.2 \mathrm{meV}$. The strong anisotropy in magnetic coupling is a direct consequence of the layered anion structure since the electronic states at the top of the valence band are comprised almost exclusively of $3 \mathrm{C} \mathrm{O} p$ character [51,67]. Importantly even within the $3 \mathrm{C}$ oxygen layers the magnetic coupling between $\mathrm{Hf}$ vacancy defects is far weaker than is obtained using a standard semilocal exchange correlation functional which is a result of the much increased localization (e.g., applying GGA in this case gives

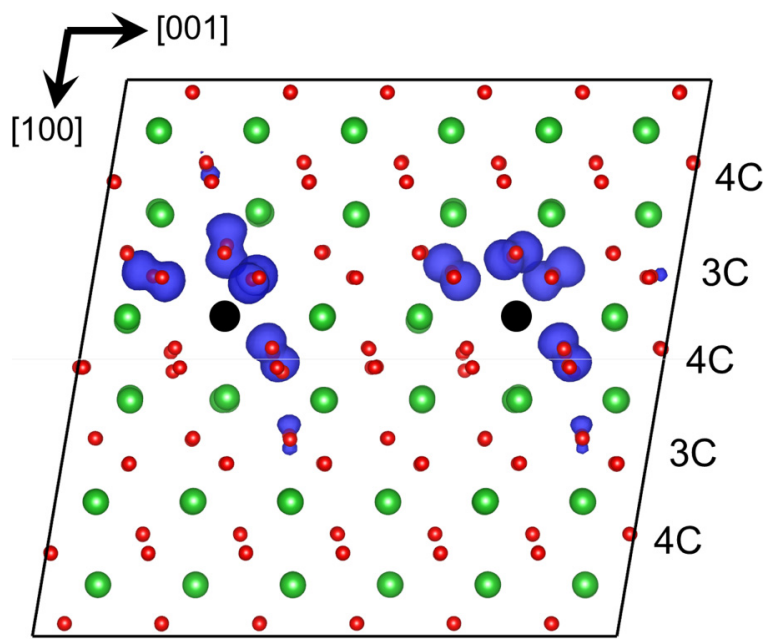

FIG. 6. (Color online) Two $\mathrm{V}_{\mathrm{Hf}}^{X}$ defects forming a onedimensional chain in the [001] direction. Large green spheres and small red spheres represent $\mathrm{Hf}$ and $\mathrm{O}$ ions, respectively, and the black circles indicate the location of the missing Hf ions. Electron spin density isosurfaces for the case of ferromagnetic alignment are shown in blue.
$J=28 \mathrm{meV}$ ). As a result of the weak coupling, cation vacancy related ferromagnetism would only be expected at very high defect concentrations. For example, considering an effective two-dimensional Ising square lattice model we can estimate the Curie temperature using the analytic formula $T_{\mathrm{c}}=2 J / k_{\mathrm{B}} \ln (1+\sqrt{2})$ [68]. For $J=10 \mathrm{meV}$ we predict $T_{c}=263 \mathrm{~K}$ for a very high defect concentration of the order of $10^{21} \mathrm{~cm}^{-3}$.

\section{DISCUSSION}

The CON approach employed here employs a correcting potential [Eq. (1)] to eliminate the SI error in the PBE functional. The only free parameter in this potential was fitted previously to ensure linearity of the total energy with electron occupation number for small hole polarons in $m-\mathrm{HfO}_{2}$ [51]. In applying this approach to model cation vacancies, we have checked the linearity of the functional by comparing the vertical CTLs with the energy of the unoccupied one-electron eigenstates. For all defect charge states these two quantities are equal to within $0.1 \mathrm{eV}$ indicating the Koopmans condition is very close to being satisfied across the entire range of defect charge states. Therefore, we conservatively estimate that this approach is able to predict CTLs with an accuracy of the order of $0.2 \mathrm{eV}$. The consistency between the CTLs obtained with the nonlocal HSE functional and CON is further confirmation that the approach is accurate. The fact that the calculated density of states and spin density indicates holes are removed one-by-one by electron addition is a good indication that the CON approach is SI free. This is not found to be the case in the GGA calculations, for example, which delocalize the hole across several anions due to SI error. On the other hand, the GGA functional gives a qualitatively incorrect picture of hole localization and predicts CTLs too close to the valence band maximum.

We note that the HSE calculations are performed on a smaller supercell (96 atoms versus 324 atoms) due to their high computational expense, and so results are likely to be more affected by artificial interactions between periodic defect images. The much smaller computational expense of the CON approach allowing larger supercells to be considered is an important advantage of the approach and essential for considering effects such as hole diffusion and magnetic interaction between vacancies. One disadvantage of the CON approach is that it is designed to eliminate SI specifically for holes in $\mathrm{HfO}_{2}$, therefore it cannot be transferred to other types of defects or systems without reparameterization. It is also not designed to describe the oxygen molecule, and application of the CON method leads to a significant underbinding of the oxygen molecule. Importantly, this has no effect on the predicted CTLs, electronic or magnetic properties which are the main focus of this study.

We have made a number of predictions regarding the electronic and magnetic properties of hafnium vacancies which merit further discussion:

(1) We predict cation vacancies are associated with a series of CTLs in the range $0.76-1.67 \mathrm{eV}$ above the valence band maximum. These energies are significantly lower than those associated with oxygen vacancies (closer to mid gap) and so should be distinguishable using techniques such 
as deep level transient spectroscopy or capacitance-voltage profiling [69]. The presence of such levels suggests cation vacancies could play an important role as charge traps with relevance to microelectronics, for example in bias temperature instability [13].

(2) While we do not attempt to calculate spectroscopic properties of the vacancies, we can make some semiquantitative predictions based on the calculated electronic structure. In particular, electronic transitions from the valence band to unoccupied hole states localized at the cation vacancy are expected for photon energies in the range 1.5-2.3 eV. This absorption band is distinct from that predicted for neutral and positively charged oxygen vacancies (2.5-4.9 eV [58]) and so could provide another tool to quantify the vacancy content $\mathrm{HfO}_{2}$ samples. For the $\mathrm{V}_{\mathrm{Hf}}^{\prime \prime \prime}$ defect both the highest occupied and lowest unoccupied electronic states are localized suggesting the transition close to $2.4 \mathrm{eV}$ would have a higher oscillator strength. The $\mathrm{V}_{\mathrm{Hf}}^{\prime \prime \prime \prime}$ defect however has no unoccupied states in the gap, and the lowest electronic transitions are expected to occur at energies similar to the bulk band gap $(>5 \mathrm{eV})$.

(3) The calculation of the binding energy between free hole polarons and cation vacancies provides an atomistic model for the experimentally observed $p$-type conductivity at high temperatures. In particular we find that the energy required to separate a hole polaron from a neutral hafnium vacancy is about $0.6 \mathrm{eV}$. This is consistent with the activation energies extracted from measurements of $p$-type conductivity at high temperature in $\mathrm{HfO}_{2}$ [28,29]. However, we predict liberating additional holes becomes increasingly more difficult suggesting that each vacancy provides on average one hole carrier rather than the four that might be expected on the basis of formal ionic charge.

(4) We find that cation vacancies are associated with a net magnetic moment in agreement with previous studies. We predict ferromagnetic coupling between separated vacancies, which is highly anisotropic, being stronger when mediated by holes localized in the same $3 \mathrm{C}$ oxygen layer. However, the strength of the coupling is weak due to the strong hole localization, suggesting cation vacancies are an unlikely candidate to explain the unexpected ferromagnetism observed in $\mathrm{HfO}_{2}$. Only if very high concentrations of vacancies (corresponding to defect separations of the order of $10 \AA$ ) can be realized, e.g., by segregation to two-dimensional defects such as surfaces or grain boundaries, could hafnium vacancies realistically contribute to the observed ferromagnetism. We also calculate the ESR $g$ tensors associated with cation vacancies which we find are quite distinct from those due to other intrinsic defects in $\mathrm{HfO}_{2}$ providing an additional experimental route to defect identification.

\section{CONCLUSIONS}

In summary, we have performed a first principles investigation into the electronic and magnetic properties of hafnium vacancies in $m-\mathrm{HfO}_{2}$. We show how widely used semilocal approximations to exchange and correlation describe these defects in a qualitatively incorrect way, predicting hole states which are too shallow due to the self-interaction error. To obtain more accurate predictions we employ a self-interaction corrected approach which ensures linearity of the total energy with electron occupation number. We predict a series of charge transition levels in the range $0.76-1.67 \mathrm{eV}$ above the valence band maximum connected to small hole polaron states localized on oxygen ions neighboring the cation vacancy. These holes are magnetically aligned giving rise to a net magnetic moment of up to $4 \mu_{\mathrm{B}}$. However we find the magnetic coupling between separated vacancies is extremely weak, suggesting they are unlikely to give rise to a ferromagnetic state. We show how small hole polarons can be detached from cation vacancies with activation energies consistent with high temperature $p$-type conductivity measurements [28-30]. We also show how the spectroscopic properties of cation vacancies are distinct from that due to other intrinsic defects, suggesting techniques such as optical absorption spectroscopy and electron spin spectroscopy could provide a means to quantitative defect characterization. The predicted charge trapping properties of cation vacancies suggest they could play a role in microelectronics, where $\mathrm{HfO}_{2}$ is widely used as a gate dielectric and as the active layer in resistive switching memories $[13,70,71]$. While this investigation has focused on $m-\mathrm{HfO}_{2}$, very similar properties are expected for $m-\mathrm{ZrO}_{2}$, which finds a wide range of different applications [72,73]. Altogether these predictions provide detailed insight into the properties of cation vacancies in $\mathrm{HfO}_{2}$ and will be invaluable to help interpret results of experimental characterization.

\section{ACKNOWLEDGMENTS}

We acknowledge useful discussions with Alex Shluger, Stephan Lany, and Alex Zunger. K.P.M. acknowledges support from EPSRC (EP/K003151). This work made use of the facilities of Archer, the UK's national high-performance computing service, via our membership in the UK HPC Materials Chemistry Consortium, which is funded by EPSRC (EP/L000202). We also gratefully acknowledge computational resources provided by WPI Advanced Institute for Materials Research, Tohoku University, Japan.

All data created during this research are available by request from the University of York Research database [74].
[1] S. K. Deb, Philos. Mag. 27, 801 (1973).

[2] B. Henderson, Crit. Rev. Solid State Mater. Sci. 9, 1 (1980).

[3] A. M. Stoneham, Theory of Defects in Solids: Electronic Structure of Defects in Insulators and Semiconductors (Oxford University Press, Oxford, 2001).

[4] S. Samson and C. G. Fonstad, J. Appl. Phys. 44, 4618 (1973).

[5] G. Frank and H. Köstlin, Appl. Phys. A 27, 197 (1982).
[6] D. E. Williams, Sens. Actuators, B 57, 1 (1999).

[7] S. Lany and A. Zunger, Phys. Rev. Lett. 98, 045501 (2007).

[8] P. Ágoston, K. Albe, R. M. Nieminen, and M. J. Puska, Phys. Rev. Lett. 103, 245501 (2009).

[9] K. Xiong, J. Robertson, M. C. Gibson, and S. J. Clark, Appl. Phys. Lett. 87, 183505 (2005). 
[10] P. Broqvist and A. Pasquarello, Appl. Phys. Lett. 89, 262904 (2006).

[11] J. L. Gavartin, D. Muñoz Ramo, A. L. Shluger, G. Bersuker, and B. H. Lee, Appl. Phys. Lett. 89, 082908 (2006).

[12] K. Tse, D. Liu, K. Xiong, and J. Robertson, Microelectron. Eng. 84, 2028 (2007).

[13] T. Grasser, B. Kaczer, W. Goes, H. Reisinger, T. Aichinger, P. Hehenberger, P.-J. Wagner, F. Schanovsky, J. Franco, M. Luque et al., IEEE T. Electron Dev. 58, 3652 (2011).

[14] K. Vanheusden, W. L. Warren, C. H. Seager, D. R. Tallant, J. A. Voigt, and B. E. Gnade, J. Appl. Phys. 79, 7983 (1996).

[15] T.-H. Xie and J. Lin, J. Phys. Chem. C 111, 9968 (2007).

[16] J. Wang, D. N. Tafen, J. P. Lewis, Z. Hong, A. Manivannan, M. Zhi, M. Li, and N. Wu, J. Am. Chem. Soc. 131, 12290 (2009).

[17] B. H. Rose and D. L. Cowan, Solid State Commun. 15, 775 (1974).

[18] B. H. Rose and L. E. Halliburton, J. Phys. C: Solid State 7, 3981 (1974).

[19] B. Henderson, J. Phys. C: Solid State 9, L579 (1976).

[20] O. F. Schirmer, J. Phys.: Condens. Matter 18, R667 (2006).

[21] H. Raebiger, S. Lany, and A. Zunger, Phys. Rev. B 76, 045209 (2007).

[22] D. J. Keeble, S. Wicklein, R. Dittmann, L. Ravelli, R. A. Mackie, and W. Egger, Phys. Rev. Lett. 105, 226102 (2010).

[23] A. Droghetti, C. D. Pemmaraju, and S. Sanvito, Phys. Rev. B 81, 092403 (2010).

[24] J. P. Perdew and A. Zunger, Phys. Rev. B 23, 5048 (1981).

[25] S. Lany and A. Zunger, Phys. Rev. B 80, 085202 (2009).

[26] J. Robertson, Rep. Prog. Phys. 69, 327 (2006).

[27] M. Y. Chan, T. Zhang, V. Ho, and P. S. Lee, Microelectron. Eng. 85, 2420 (2008).

[28] R. W. Vest, N. M. Tallan, and W. C. Tripp, J. Am. Ceram. Soc. 47, 635 (1964).

[29] N. M. Tallan, W. C. Tripp, and R. W. Vest, J. Am. Ceram. Soc. 50, 279 (1967).

[30] C. Ko, M. Shandalov, P. C. McIntyre, and S. Ramanathan, Appl. Phys. Lett. 97, 082102 (2010).

[31] M. Venkatesan, C. B. Fitzgerald, and J. M. D. Coey, Nature (London) 430, 630 (2004).

[32] J. M. D. Coey, M. Venkatesan, P. Stamenov, C. B. Fitzgerald, and L. S. Dorneles, Phys. Rev. B 72, 024450 (2005).

[33] G. M. E. Tirosh, Adv. Mater. 19, 2608 (2007).

[34] J. Osorio-Guillén, S. Lany, S. V. Barabash, and A. Zunger, Phys. Rev. B 75, 184421 (2007).

[35] K. K. Bharathi, S. Venkatesh, G. Prathiba, N. H. Kumar, and C. V. Ramana, J. Appl. Phys. 109, 07C318 (2011).

[36] A. J. Tench and M. J. Duck, J. Phys. C: Solid State 8, 257 (1975).

[37] P. Kofstadt, Non-Stoichiometry, Diffusion, and Electrical Conductivity in Binary Metal Oxides (Wiley, New York, 1972).

[38] F. Tuomisto, V. Ranki, K. Saarinen, and D. C. Look, Phys. Rev. Lett. 91, 205502 (2003).

[39] S. Zhang, S. B. Ogale, W. Yu, X. Gao, T. Liu, S. Ghosh, G. P. Das, A. T. S. Wee, R. L. Greene, and T. Venkatesan, Adv. Mater. 21, 2282 (2009).

[40] J. Mizusaki, N. Mori, H. Takai, Y. Yonemura, H. Minamiue, H. Tagawa, M. Dokiya, H. Inaba, K. Naraya, T. Sasamoto et al., Solid State Ionics 129, 163 (2000).

[41] I. S. Elfimov, S. Yunoki, and G. A. Sawatzky, Phys. Rev. Lett. 89, 216403 (2002).
[42] J. Beltrán, C. Monty, L. Balcells, and C. Martínez-Boubeta, Solid State Commun. 149, 1654 (2009).

[43] F. Gao, J. Hu, C. Yang, Y. Zheng, H. Qin, L. Sun, X. Kong, and M. Jiang, Solid State Commun. 149, 855 (2009).

[44] M. Cococcioni and S. de Gironcoli, Phys. Rev. B 71, 035105 (2005).

[45] S. Lany and A. Zunger, Phys. Rev. B 81, 205209 (2010).

[46] J. Heyd, G. E. Scuseria, and M. Ernzerhof, J. Chem. Phys. 118, 8207 (2003).

[47] D. Muñoz Ramo, A. L. Shluger, J. L. Gavartin, and G. Bersuker, Phys. Rev. Lett. 99, 155504 (2007).

[48] D. O. Scanlon, B. J. Morgan, G. W. Watson, and A. Walsh, Phys. Rev. Lett. 103, 096405 (2009).

[49] F. Oba, M. Choi, A. Togo, and I. Tanaka, Sci. Technol. Adv. Mater. 12, 034302 (2011).

[50] T. R. Paudel, A. Zakutayev, S. Lany, M. d'Avezac, and A. Zunger, Adv. Func. Mater. 21, 4493 (2011).

[51] K. P. McKenna, M. J. Wolf, A. L. Shluger, S. Lany, and A. Zunger, Phys. Rev. Lett. 108, 116403 (2012).

[52] C. Das Pemmaraju and S. Sanvito, Phys. Rev. Lett. 94, 217205 (2005).

[53] G. Kresse and J. Furthmüller, Phys. Rev. B 54, 11169 (1996).

[54] G. Kresse and J. Furthmüller, J. Comput. Mater. 6, 15 (1996).

[55] M. J. Wolf, K. P. McKenna, and A. L. Shluger, J. Phys. Chem. C 116, 25888 (2012).

[56] S. Lany, H. Raebiger, and A. Zunger, Phys. Rev. B 77, 241201 (2008).

[57] S. Lany, Phys. Status Solidi B 248, 1052 (2011).

[58] D. Muñoz Ramo, J. L. Gavartin, A. L. Shluger, and G. Bersuker, Phys. Rev. B 75, 205336 (2007).

[59] P. V. Sushko, A. L. Shluger, and C. R. A. Catlow, Surf. Sci. 450, 153 (2000).

[60] D. Muñoz Ramo, A. L. Shluger, and G. Bersuker, Phys. Rev. B 79, 035306 (2009).

[61] D. Muñoz Ramo, P. V. Sushko, and A. L. Shluger, Phys. Rev. B 85, 024120 (2012).

[62] M. J. Frisch et al., GAUSSIAN 03 Revision CO2 (Gaussian Inc., Wallingford, CT, 2003).

[63] P. J. Hay and W. R. Wadt, J. Chem. Phys. 82, 270 (1985).

[64] J. Carrasco, C. Sousa, F. Illas, P. V. Sushko, and A. L. Shluger, J. Chem. Phys. 125, 074710 (2006).

[65] http://www.crystal.unito.it/Basis_Sets/Ptable.html.

[66] C. Loschen, J. Carrasco, K. M. Neyman, and F. Illas, Phys. Rev. B 75, 035115 (2007).

[67] J. E. Medvedeva, A. J. Freeman, C. B. Geller, and D. M. Rishel, Phys. Rev. B 76, 235115 (2007).

[68] L. Onsager, Phys. Rev. 65, 117 (1944).

[69] S. Dueñas, H. Castán, J. Barbolla, K. Kukli, M. Ritala, and M. Leskelä, Solid-State Electron. 47, 1623 (2003).

[70] W.-T. Lu, P.-C. Lin, T.-Y. Huang, C.-H. Chien, M.-J. Yang, I.-J. Huang, and P. Lehnen, Appl. Phys. Lett. 85, 3525 (2004).

[71] J. A. Rothschild, H. Avraham, E. Lipp, and M. Eizenberg, Appl. Phys. Lett. 96, 122102 (2010).

[72] J.-H. Lee, J. Mater. Sci. 38, 4247 (2003).

[73] P. F. Manicone, P. R. Iommetti, and L. Raffaelli, J. Dent. (Oxford, U. K.) 35, 819 (2007).

[74] http://dx.doi.org/10.15124/54729b94-5d5a-4831-a15d04ab3f617298. 